Cite this: Phys. Chem. Chem. Phys., 2012, 14, 6332-6342

\title{
Tracking energy transfer from excited to accepting modes: application to water bend vibrational relaxation $\dagger$
}

\author{
Rossend Rey ${ }^{* a}$ and James T. Hynes ${ }^{b c}$ \\ Received 11th November 2011, Accepted 20th February 2012 \\ DOI: $10.1039 / \mathrm{c2cp23555b}$
}

We extend, via a reformulation in terms of Poisson brackets, the method developed previously (Rey et al., J. Phys. Chem. A, 2009, 113, 8949) allowing analysis of the pathways of an excited molecule's ultrafast vibrational relaxation in terms of intramolecular and intermolecular contributions. In particular we show how to ascertain, through the computation of power and work, which portion of an initial excess molecular energy (e.g. vibrational) is transferred to various degrees of freedom (e.g. rotational, translational) of the excited molecule itself and its neighbors. The particular case of bend excess energy relaxation in pure water is treated in detail, completing the picture reported in the work cited above. It is shown explicitly, within a classical description, that almost all of the initial water bend excitation energy is transferred - either indirectly, via Fermi resonance centrifugal coupling to the bend-excited water's rotation, or directly via intermolecular coupling - to local water librations, only involving molecules in the first two hydration shells of the vibrationally excited water molecule. Finally, it is pointed out that the Poisson bracket formulation can also be applied to elucidate the microscopic character of solvation and rotational dynamics, and should prove useful in developing a quantum treatment for energy flow in condensed phases.

\section{Introduction}

Vibrational relaxation of small molecules in the liquid phase has a long history, ${ }^{1-7}$ with considerable progress during the last several decades fueled by the interplay of numerical simulations and time-dependent spectroscopy. ${ }^{8-11}$ Water has naturally attracted special interest in this regard. Initial efforts here focused, for both theoretical and technical reasons, on vibrational relaxation of the stretches in isotopically substituted water molecules, and an extensive literature is available. ${ }^{8,12}$

Ultrafast spectroscopy advances have shifted the focus in the last few years towards pure water. Among other advances, experiments have been able to address energy (population) relaxation of the water bend vibration which is generally regarded as the last step of the vibrational relaxation ladder, ${ }^{13}$ and (high frequency) librational i.e. hindered rotation relaxation. Population relaxation times are extremely short in both cases, approximately $200 \mathrm{fs}$ for the bend and less than $50 \mathrm{fs}$ for librations. ${ }^{14}$ In this limit, the dynamics is well described by a

${ }^{a}$ Departament de Física i Enginyeria Nuclear, Universitat Politècnica de Catalunya, Campus Nord B4-B5, Barcelona 08034, Spain.

E-mail: rosendo.rey@upc.edu

${ }^{b}$ Department of Chemistry and Biochemistry University of Colorado, Boulder,CO 80309-0215,USA.E-mail: hynes@spot.colorado.edu

${ }^{c}$ Chemistry Department, Ecole Normale Supérieure,

CNRS UMR-8640, 24 Rue Lhomond, 75005 Paris, France

$\dagger$ Electronic supplementary information (ESI) available. See DOI: $10.1039 / \mathrm{c} 2 \mathrm{cp} 23555 \mathrm{~b}$ harmonic approximation, ${ }^{15}$ which combined with the equivalence between harmonic classical and quantum dynamics, suggests that a classical description of these processes should constitute a reasonable approach. Indeed, in the first theoretical studies of bend relaxation ${ }^{16,17}$ good agreement was found between experimental results and fully classical simulations.

Of course, interest lies not just in reproducing the experimental time, but also in obtaining information, as yet not experimentally accessible, on the relaxation and energy transfer pathways. To this end, classical simulations are particularly convenient. In principle, the simplest, direct way to address such mechanistic issues is to track the different contributions to the system's total energy during a nonequilibrium simulation, with the hope that from their time evolution a picture of the relaxation pathways will emerge. Unfortunately, this approach is subject to important difficulties if, for instance, the accepting modes relax on a time scale considerably shorter than that of the initially excited mode, as occurs for water bending relaxation. ${ }^{16}$ There is then little or no significant buildup of energy in these modes so that, even though they are actually channeling the excess energy, this energy flow's footprint is extremely feeble. As a result, the fit of observed time dependent excess energy evolution with phenomenological kinetic equations ${ }^{17}$ is subject to considerable ambiguities, as is any indirectly inferred energy transfer mechanism, since it is not directly addressed.

It was shown in ref. 17 how these difficulties could be circumvented by building on previous work on vibrational 
relaxation of diatomics. ${ }^{18}$ Basically, one first computes the energy derivative (power), followed by its integral (work). The mathematical structure of power (force times velocity) allows one to pinpoint which molecule or mode is channeling energy from the excited molecular mode to/from the mode of interest, since the force can be partitioned in contributions from different neighbors and velocities can be ascribed to particular modes of motion. After integration (work), one can determine unambiguously the total energy transferred to each neighbor or internal mode. This approach was applied to water bend relaxation, ${ }^{17}$ and it was shown that the main pathway is intramolecular transfer via 2:1 Fermi resonance to self(hindered) rotation mediated by the centrifugal coupling mechanism. With the computation of the power exerted by external water molecules it was possible to determine, for instance, that the portion of energy directly transferred to its neighbors (that is, the part not transferred to internal rotation) was mainly taken up by the two water molecules with their oxygens hydrogen-bonded to the excited water molecule.

However, an important issue not addressed was the identity of the specific accepting modes within the neighboring water molecules. Thus, it was not determined whether this energy was transferred to potential energy of interaction, hindered rotation of each neighbor, or neighboring water translation. It is the purpose of the present work to elucidate these aspects and, to this end, we include a detailed discussion of how the expressions for power and work can be manipulated very conveniently via a Poisson bracket formulation to yield the desired information.

The organization of the remainder of this paper is as follows. In Section II, we present the basic theoretical Poisson bracket formulation. Application to bend relaxation in pure liquid water is the subject of Section III. Concluding remarks are offered in Section IV. Several extensions of the formulation are given in an Appendix.

\section{Theory}

Here we derive the formulas required to compute the excited molecule's energy transferred to the degrees of freedom of choice. We show that the Poisson bracket formulation of classical mechanics is particularly transparent in this connection and, as a bonus, sets the stage for a future extension to a quantum formalism. The first subsection is devoted to rewriting the equations developed in ref. 17 for the relaxation of water bending in terms of this language. In the following subsection these equations are extended in order to pinpoint the specific modes to which energy is transferred. The main formulas for bending relaxation are then summarized.

\section{A. Power and Poisson brackets}

We commence with the standard Hamiltonian formulation in terms of a portion corresponding to the system of interest ( $H_{1}$, which can be subpartitioned if necessary) plus its coupling ( $V$, here assumed to be purely configurational) to the environment $\left(\mathrm{H}_{2}\right)$

$$
H=H_{1}+V+H_{2}
$$

The energy flux (power) is the time derivative of $H_{1}$ which, as for any dynamic variable not explicitly time-dependent as assumed here, can be expressed in terms of Poisson brackets ${ }^{19}$

$$
P_{1} \equiv \frac{\mathrm{d} H_{1}}{\mathrm{~d} t}=\left[H_{1}, H\right]
$$

Before dealing with the water bend problem it is useful to treat several much simpler problems first, for purposes of orientation and for subsequent employment in the bend energy flow treatment. A simplest application is that of a system of point particles in three dimensions. If $H_{1}$ represents the energy of a given particle (purely kinetic, $H_{1}=K_{1}$ ), then

$$
\frac{\mathrm{d} H_{1}}{\mathrm{~d} t}=\left[H_{1}, H\right]=\left[K_{1}, V\right]=-\frac{\partial K_{1}}{\partial \vec{p}_{1}} \cdot \frac{\partial V}{\partial \vec{r}_{1}}=\vec{v}_{1} \cdot \vec{F}_{1},
$$

i.e. the standard textbook formula. Since $\vec{F}_{1}$ (external force on particle 1) can be partitioned in terms of the various forces exerted on it $\left(\vec{F}_{1}=\sum_{i>1} \vec{F}_{i 1}\right)$, it is straightforward to track the contribution of each neighbor. It is this additive property which lends most of its utility to the present approach, as we seek to pinpoint the specific pathways through which excess energy is transferred.

In practice, one is interested in the time variation of this excess energy (here $K_{1}(t)-K_{1}(0)$ ) so that, instead of power, the integrated form (work) is more convenient

$$
\Delta K_{1}(t) \equiv K_{1}(t)-K_{1}(0)=\int_{0}^{t} \vec{F}_{1} \cdot \vec{v}_{1} \mathrm{~d} t \equiv W_{1}^{\text {solvent }}=\sum_{i>1} W_{i 1},
$$

where, again, the last term reflects the fact that work on particle 1 ( $\left.W_{1}^{\text {solvent }}\right)$ results from the contribution of each neighbor $\left(W_{i 1}\right)$.

A second application is that of rotational relaxation for a system of rigid molecules. In this case we identify $H_{1}=K_{1}^{\mathrm{R}}$ (i.e. rotational energy of molecule 1 ), while $H_{2}$ will contain the total kinetic energy of the neighbors plus translational energy of the rotationally excited molecule. With $\left\{\vec{r}_{i}\right\}$ denoting the position vectors of the excited molecule's atoms, we have $^{19}$ (summation over repeated indexes will be adopted throughout)

$$
\begin{aligned}
\frac{\mathrm{d} H_{1}}{\mathrm{~d} t} & =\left[K_{1}^{\mathrm{R}}, H\right]=\left[K_{1}^{\mathrm{R}}, V\right]=-\frac{\partial K_{1}^{\mathrm{R}}}{\partial \vec{p}_{i}} \cdot \frac{\partial V}{\partial \vec{r}_{i}} \\
& =\left(\vec{\omega} \times \vec{r}_{i}\right) \cdot \vec{F}_{i}=\vec{\omega} \cdot \vec{\tau},
\end{aligned}
$$

i.e. the well-known torque times angular velocity formula.

As the main object of this subsection, we rederive the formulas for relaxation of water bending (obtained in ref. 17) from the different, Poisson bracket perspective. Our model is that of a water molecule with fixed oxygen-hydrogen bond lengths and variable bending angle, summarized in the Hamiltonian ${ }^{20}$

$$
\begin{aligned}
H_{\mathrm{mol}}=K_{\mathrm{T}}+K_{\mathrm{R}}+H_{\mathrm{v}} \equiv & \frac{1}{2} M \vec{v}_{\mathrm{CM}}^{2}+\frac{1}{2} m_{i}\left(\vec{\omega} \times \vec{r}_{i}\right)^{2} \\
& +\frac{1}{2} m_{i} \vec{v}_{i}^{2}+U(\theta),
\end{aligned}
$$

i.e. center of mass (CM) kinetic energy, plus rotational kinetic energy, plus vibrational energy, which comprises the last two terms. Coriolis coupling cancels out for this model, ${ }^{17}$ and therefore what follows is valid for diatomic molecules as well. 
We make the identification $H_{1}=H_{\mathrm{v}}$, while $H_{2}$ will contain the rest of the molecules plus rotation and translational energy of the excited molecule. Applying again eqn (2) we obtain

$$
\frac{\mathrm{d} H_{\mathrm{v}}}{\mathrm{d} t}=\left[H_{\mathrm{v}}, H\right]=\left[H_{\mathrm{v}}, K_{\mathrm{R}}\right]+\left[H_{\mathrm{v}}, V\right],
$$

where the only two surviving terms are easily interpretable: the first one $\left(\left[H_{\mathrm{v}}, K_{\mathrm{R}}\right]\right)$ corresponds to centrifugal coupling, while the second one $\left(\left[H_{\mathrm{v}}, V\right]\right)$ represents the effect of external forces on vibrational motion (the only contribution for point particles, see eqn (3)), i.e.

$$
\left[H_{\mathrm{v}}, V\right]=-\frac{\partial H_{\mathrm{v}}}{\partial p} \frac{\partial V}{\partial q}=-\dot{q} \frac{\partial V}{\partial q}
$$

where $q$ denotes the bending degree of freedom (with generalized momentum $p$ ). It can be written as well in terms of the forces on each of the atoms (denoted by index $i$ ) in the central water molecule,

$$
\left[H_{\mathrm{v}}, V\right]=-\dot{q} \frac{\partial V}{\partial q}=-\dot{q} \frac{\partial V}{\partial \vec{r}_{i}} \cdot \frac{\partial \vec{r}_{i}}{\partial q}=\vec{F}_{i} \cdot \vec{v}_{i},
$$

in which velocities are defined as $\vec{v}_{i} \equiv \frac{\partial \vec{r}_{i}}{\partial q} \dot{q}$, and therefore correspond to the moving molecular frame, the Eckart frame. ${ }^{21}$

We can also expand the centrifugal coupling term in eqn (7) to recover the formulas given in ref. 17 . We recall that

$$
K_{\mathrm{R}}=\frac{1}{2} I_{i} \omega_{i}^{2},
$$

so that

$$
\left[H_{\mathrm{v}}, H_{\mathrm{R}}\right]=-\frac{\partial H_{\mathrm{v}}}{\partial p} \frac{\partial K_{\mathrm{R}}}{\partial q}=-\frac{1}{2} \dot{q} \frac{\partial I_{i}}{\partial q} \omega_{i}^{2},
$$

which shows the Fermi coupling ${ }^{17}$ between vibration (term linear in $\dot{q}$ ) and rotation (quadratic dependence on angular velocity) referred to in the Introduction. Finally, we obtain for the vibrational energy derivative

$$
\frac{\mathrm{d} H_{\mathrm{v}}}{\mathrm{d} t}=\left[H_{\mathrm{v}}, K_{\mathrm{R}}\right]+\left[H_{\mathrm{v}}, V\right]=\vec{F}_{i} \cdot \vec{v}_{i}-\frac{1}{2} \dot{q} \frac{\partial I_{i}}{\partial q} \omega_{i}^{2},
$$

whose first and final components represent the expression used in our previous work. ${ }^{17}$

\section{B. Transfer to specific modes of surrounding molecules}

The formulas developed thus far will allow us to ascertain to which internal mode(s) of the excited molecule and to which neighbouring molecules energy is being transferred. In addition, one would like to know-and this our main, novel goal - the specific accepting mode (i.e. rotation, translation, or other modes) on the neighboring molecules. In the following, we proceed in a general fashion, pausing at various points to illustrate some of the key ideas and relations.

The time evolution of the potential energy of interaction plays a crucial role in mediating energy transfer. ${ }^{22}$ One can track its involvement by computing its time derivative, much in the same way as was done in eqn (2) for $H_{1}$,

$$
\frac{\mathrm{d} V}{\mathrm{~d} t}=[V, H]=\left[V, H_{1}\right]+\left[V, H_{2}\right],
$$

so that

$$
\left[H_{1}, V\right]=-\frac{\mathrm{d} V}{\mathrm{~d} t}+\left[V, H_{2}\right]
$$

The utility of this relation can be illustrated at the outset with the point particle in 3 dimensions case with $H_{1}=K_{1}$. Using eqn (14) in conjunction with eqn (3), and noting the change in sign upon transposition of the members of a Poisson bracket, we obtain

$$
\begin{aligned}
\frac{\mathrm{d} H_{1}}{\mathrm{~d} t} & =\left[H_{1}, V\right]=-\frac{\mathrm{d} V}{\mathrm{~d} t}-\left[H_{2}, V\right]=-\frac{\mathrm{d} V}{\mathrm{~d} t}+\frac{\partial H_{2}}{\partial \vec{p}_{i}} \cdot \frac{\partial V}{\partial \vec{r}_{i}} \\
& =-\frac{\mathrm{d} V}{\mathrm{~d} t}-\vec{v}_{i} \cdot \vec{F}_{1 i} .
\end{aligned}
$$

The integral form reads

$$
\Delta K_{1}(t)=-\Delta V(t)-\sum_{i>1} W_{1 i}
$$

which summarizes the basic idea: a change in the initially excited particle's kinetic energy is turned into a potential energy of interaction plus a work on each one of its neighbors (note that this work differs from that in eqn (4): now it is exerted by particle 1 on particle $i, W_{1 i}$, as opposed to the work of $i$ on $\left.1, W_{i 1}\right)$. One can think in terms of a liquid at equilibrium, and where particle 1 is subjected to an initial kick: since equilibrium will be reached again after a transient, we will have (after a thermal average) $\langle\Delta V(t)\rangle \rightarrow 0$ and, therefore, only the sum over the work terms will survive. From this sum it will be possible to extract which percentages of the initial kinetic energy are channeled (on the average) through each neighbor.

We now extend this approach to include the vibrational degree of freedom described at the end of Section 2. To this end, it is convenient to consider first the entire molecule and then extract the vibrational energy behaviour. Taking $H_{1}=H_{\mathrm{mol}}$, the molecular Hamiltonian in eqn (6), and applying the same approach as for the point particle case (see eqn (13) and (14)), we find

$$
\frac{\mathrm{d} H_{\mathrm{mol}}}{\mathrm{d} t}=\left[H_{\mathrm{mol}}, H\right]=\left[H_{\mathrm{mol}}, V\right]=-\frac{\mathrm{d} V}{\mathrm{~d} t}-\left[H_{2}, V\right],
$$

where $\mathrm{H}_{2}$ contains only the molecule's solvent neighbors, and therefore we can write

$$
\left[H_{2}, V\right]=-\frac{\partial H_{2}}{\partial p_{j}} \frac{\partial V}{\partial q_{j}}=\dot{q}_{j} F_{1 j} \equiv \sum_{j} P_{1 j}
$$

involving a sum of powers where $F_{1 j}$ denotes the force of molecule 1 on mode $j$ of the solvent molecules external to that molecule. This power can be expressed in terms of different modes, of which a most natural one is as a sum over each molecule $j$, i.e.

$$
\left[H_{2}, V\right]=\sum_{j>1} P_{1 j}=\sum_{j>1} \sum_{j_{\mathrm{a}}} \vec{v}_{j_{\mathrm{a}}} \cdot \vec{F}_{1 j_{\mathrm{a}}}
$$

with $j_{\mathrm{a}}$ denoting the atoms in molecule $j$, and $\vec{F}_{1 j_{\mathrm{a}}}$ the total force of molecule 1 on atom $j_{\mathrm{a}}$.

In its integrated form eqn (17) can be written

$$
\Delta E_{\mathrm{mol}}(t)=-\Delta V(t)-\sum_{j>1} W_{1 j}
$$


in which the last term is the time integral of the last term of eqn (19). Eqn (20) is formally identical to eqn (16), although here the term on the left side $\left(\Delta E_{\mathrm{mol}}\right)$ contains contributions from all internal degrees of freedom of the excited molecule. Indeed, if it is expanded into its constituent terms, and the potential energy increment is transferred to the left side, we obtain

$$
\Delta K_{\mathrm{T}}+\Delta K_{\mathrm{R}}+\Delta E_{\mathrm{v}}+\Delta V=-\sum_{j>1} W_{1 j}
$$

which is a central formula of this paper. Section III will be devoted to its application to an initial excitation of the water bend, for which we rearrange it as

$$
\Delta E_{\mathrm{v}}=-\Delta V-\Delta K_{\mathrm{T}}-\Delta K_{\mathrm{R}}-\sum_{j>1} W_{1 j},
$$

where the first three terms on the right will tend to zero after a short transient (similar to $\Delta K_{\mathrm{T}}$ in the point particle case), while the last term will allow for the analysis of relaxation pathways to the excited molecule's neighbors. Before addressing that aspect, we remark that similar rearrangements can be used to address energy flow resulting from other molecular excitations, e.g. rotational and electronic, to be discussed in Section IV.

It remains to show how the work can be partitioned into different modes of the molecules surrounding the central molecule of interest. A most natural choice for the accepting modes is that of translations plus rotations of the excited molecule's neighboring molecules, although this does not preclude other choices. We recall from eqn (19) that each power contribution can be written as

$$
P_{1 j}=\sum_{j_{\mathrm{a}}} \vec{v}_{j_{\mathrm{a}}} \cdot \vec{F}_{1 j_{\mathrm{a}}}
$$

where the velocities are now defined in the laboratory frame, and $\vec{F}_{1 j_{\mathrm{a}}}$ is the force of molecule 1 on atom $j_{\mathrm{a}}$ (of molecule $j$ ). If for simplicity we limit ourselves to the case in which the neighboring molecules are rigid, then

$$
\vec{v}_{j_{\mathrm{a}}}=\vec{v}_{j}^{\mathrm{CM}}+\vec{\omega}_{j} \times \vec{r}_{j_{\mathrm{a}}},
$$

which when substituted in eqn (23) results in

$$
P_{1 j}=\sum_{j_{\mathrm{a}}}\left(\vec{v}_{j}^{\mathrm{CM}}+\vec{\omega}_{j} \times \vec{r}_{j_{\mathrm{a}}}\right) \cdot \vec{F}_{1 j_{\mathrm{a}}}=\vec{v}_{j}^{\mathrm{CM}} \cdot \vec{F}_{1 j}+\vec{\omega}_{j} \cdot \vec{\tau}_{1 j} \equiv P_{1 j}^{\mathrm{T}}+P_{1 j}^{\mathrm{R}},
$$

i.e. the sum of the power of molecule 1 on translation of molecule $j\left(P_{1 j}^{\mathrm{T}}\right)$, plus the corresponding power on rotation $\left(P_{1 j}^{\mathrm{R}}\right)$.

\section{Bend vibrational relaxation}

We can now write down the full expression that will be used in Section III to study bend relaxation. We simply need to integrate eqn (25) and substitute into eqn (22), to find

$$
\Delta E_{\mathrm{v}}=-\Delta V-\Delta K_{\mathrm{T}}-\Delta K_{\mathrm{R}}-\sum_{j>1}\left(W_{1 j}^{\mathrm{T}}+W_{1 j}^{\mathrm{R}}\right) .
$$

where the explicit expressions for the work terms are

$$
W_{1 j}^{\mathrm{T}}=\int_{0}^{t} \vec{v}_{j}^{\mathrm{CM}} \cdot \vec{F}_{1 j} \mathrm{~d} t
$$

$$
W_{1 j}^{\mathrm{R}}=\int_{0}^{t} \vec{\omega}_{j} \cdot \vec{\tau}_{1 j} \mathrm{~d} t
$$

Again, only the the sum on the right side of eqn (26) will survive, after the excited molecule has lost all its excess energy, allowing us to ascertain the modes through which energy is channeled. Alternate derivations of eqn (26), and generalizations thereof, are provided in the Appendix.

\section{Bend vibrational relaxation in liquid water}

With our key theoretical result eqn (26) in hand, we now exploit it for bend relaxation in pure liquid water, extending the results reported in ref. 16 and 17.

\section{A. Computational details}

The various molecular and simulation parameters are the same as in our previous works ${ }^{16,17}$ and we just summarize the main aspects. The rigid SPC/E model ${ }^{25}$ has been adopted for all water molecules except one, for which (in addition) bending motion has been allowed, ${ }^{16}$ keeping the oxygen-hydrogen bond lengths at a fixed value. All simulations have been run with an in-house code using 200 molecules. The time step is $1 \mathrm{fs}$, the mean temperature $300 \mathrm{~K}$, the box size is $18.179 \AA$, and the cut-off distance is half the box length. The Ewald sum correction has been included for Coulomb forces. Equations of motion have been integrated with the "RATTLE" algorithm. $^{26}$

Simulations consist of a long trajectory from which, every 2 ps, the instantaneous configuration is taken as the initial one for a separate nonequilibrium run. For each one of these nonequilibrium trajectories, the instantaneous bending angle is left unchanged and vibrational kinetic energy is added, so that a total energy of $\frac{3}{2} \hbar \omega_{\mathrm{b}}$ is placed in the bending mode, ${ }^{27}$ where $\omega_{\mathrm{b}}$ is the unperturbed harmonic bend frequency. No resampling of rotational/translational velocities is done for any of the molecules. Each nonequilibrium trajectory is run for $3 \mathrm{ps,} \mathrm{without} \mathrm{temperature} \mathrm{control,} \mathrm{while} \mathrm{the} \mathrm{quantities} \mathrm{to}$ be analyzed (energy, power, work) are computed. Given the long equilibration run required, velocities are resampled (except for the flexible molecule) right after each initial configuration (for the nonequilibrium runs) is stored (i.e. every $2 \mathrm{ps}$ ), in order to avoid potential artifacts associated with long simulation times. ${ }^{28,29}$ Temperature control is maintained ${ }^{30}$ during the equilibration runs. The results reported correspond to an average over a total of 10000 trajectories (compared to 2000 in our previous work ${ }^{17}$ ), corresponding to ten independent equilibration runs. We note that sampling of initial conditions is simpler in this work compared to ref. 17, a point to which we will return in Section III G.

\section{B. Bend energy relaxation overview}

Fig. 1 displays the core numerical results of this work, obtained after averaging each term in our central formula (26). All functions have been normalized, taking a unit value for the excess vibrational energy at the initial time.

We note first that, as reported in ref. 16 and 17, the water bend vibrational energy decays monotonically with a time 


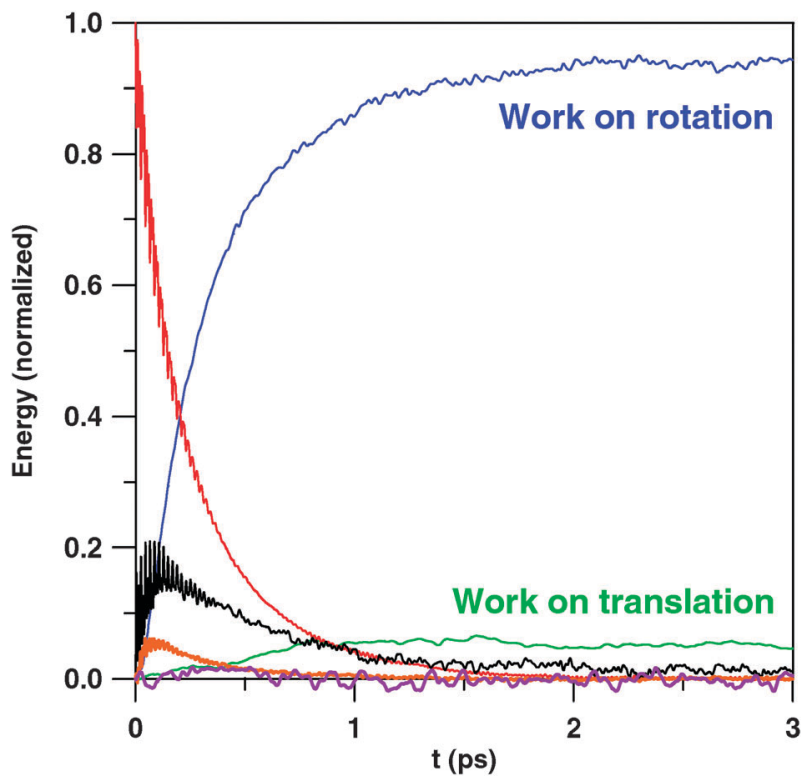

Fig. 1 Energy balance for bend relaxation in water. The functions displayed correspond to the terms in eqn (26), see Section III. Red line: $\Delta E_{v}$ (shifted upwards); black: $\Delta V$; purple: $\Delta K_{\mathrm{T}}$ for the initially excited molecule; orange: $\Delta K_{\mathrm{R}}$; green: work on translation of neighbors; blue: work on rotation of neighbors.

constant of approximately 265 fs (computed from the time integral), in reasonable accord with experimental findings $\left(170 \pm 15 \mathrm{fs}^{14,31}\right)$. The rest of the functions displayed, as described in the Theory section, can be grouped into two different classes: those that go through a transient and finally decay to zero - here the change in potential energy $(\Delta V)$, and the changes in the excited water molecule's translational $\left(\Delta K_{\mathrm{T}}\right)$ and rotational $\left(\Delta K_{\mathrm{R}}\right)$ energies - and those that reach a plateauhere the summed works $\left(\sum_{j>1} W_{1 j}^{\mathrm{T}}\right.$ and $\left.\sum_{j>1} W_{1 j}^{\mathrm{R}}\right)$ done by the excited molecule on its water neighbors, now discussed.

\section{Work contributions}

Of the two work contributions just identified, clearly the work on the hindered rotational motion of the neighboring water molecules stands out in Fig. 1. We see that the corresponding curve plateaus at approximately 0.95 , i.e. almost all the excess water bend energy is channeled through the hindered rotations of its neighbors. In our previous works this conclusion regarding the neighboring water librations was indirectly inferred: in ref. 17 it was concluded from the fit of a simplified phenomenological model to the time dependent excess energies. Another, more indirect inference was made in the related case of HOD bend relaxation in $\mathrm{D}_{2} \mathrm{O}$; a semiclassical calculation showed no differences between simulations performed with rigid or flexible molecules, so that no transfer between bendings occurred, and thus it was assumed that librations constituted the main relaxation channel. ${ }^{13}$ We believe the present demonstration is the first time in which the transfer from bending motion into rotations is shown unambiguously (stemming from such basic formula as torque times angular velocity). A detailed study in terms of the contribution of the different solvation shells will be given below.

We pause to note that Fig. 1 shows that transfer to translational motion is markedly inefficient compared to transfer to rotation, with only about $5 \%$ of the energy channeled through this mode. This result might be considered somewhat puzzling at first sight, from the perspective of energy equipartition at equilibrium, where the average rotational and translational kinetic energies for a water molecule would be equal. It is important to note, though, that these results should not be interpreted as meaning that all excess vibrational energy transferred into rotational energy stays that way. Rotational (and translational) energy relax on extremely short timescales ${ }^{16,17}$ (of the order of $50 \mathrm{fs}$ ), so that any excess energy they might receive is almost immediately transferred into other modes. The picture is one in which almost all excess energy within the excited molecule is transferred into hindered rotations of its neighbors (with the major portion of it first channeled through self-rotation of the bend-excited molecule, i.e. the process is basically one of rotational relaxation), but is immediately redistributed into progressively distant hydration shells. At each step of this process, the rotational channel prevails (a detailed investigation of rotational relaxation per se is under $w^{2} y^{23}$ ), but a portion of the energy is transferred to translations as well, so that equipartition between rotational and translational energy will be finally achieved.

\section{Transient terms}

Turning to the functions in Fig. 1 that go through a transient before vanishing, their involvement differs considerably. First, translational energy of the initially bend-excited water molecule $\left(\Delta K_{\mathrm{T}}\right)$ is apparently not involved in the process of vibrational relaxation; within the statistical indeterminacy of our calculation the results are compatible with zero. Of course, these are averaged results, so that they do not preclude the possibility that $K_{\mathrm{T}}$ 's contribution is not totally negligible for some trajectories. This result for $K_{\mathrm{T}}$ is consistent with that discussed in the preceding paragraph on transfer from rotation to translation. The only transfer to translation is to/from the excited water's neighbors, as there is no internal coupling (between rotation and translation). Since the neighboring molecules absorb energy mainly through their rotational motion, and it has been shown that transfer from rotation to translation is highly inefficient, no substantial increase in translational energy of the central molecule should be expected resulting from excited rotations on immediate neighbors.

The situation is different for the bend-excited water molecule's rotational kinetic energy $\left(\Delta K_{\mathrm{R}}\right)$ in Fig. 1, for which we observe a transient increase for times shorter than approximately $50 \mathrm{fs}$, as already reported in ref. 16 and 17. Again, the fast relaxation of this mode of motion explains the feeble maximum, reaching $\approx 10 \%$ of the total initial energy, a very modest increase considering that most of the total initial excess energy is channeled through it by the intramolecular Fermi resonance bend-rotation centrifugal coupling. ${ }^{17}$

Finally, the potential energy increment $(\Delta V)$ is the most marked of all the transient contributions, reaching up to $20 \%$ of the initial excess energy. As should be expected, a blow-up at short times, see Fig. 2, shows that $\Delta V$ has the fastest rise among all functions displayed, i.e. the very initial transfer is into potential energy, which results in a subsequent transfer into the other intermolecular modes, precluding any further 


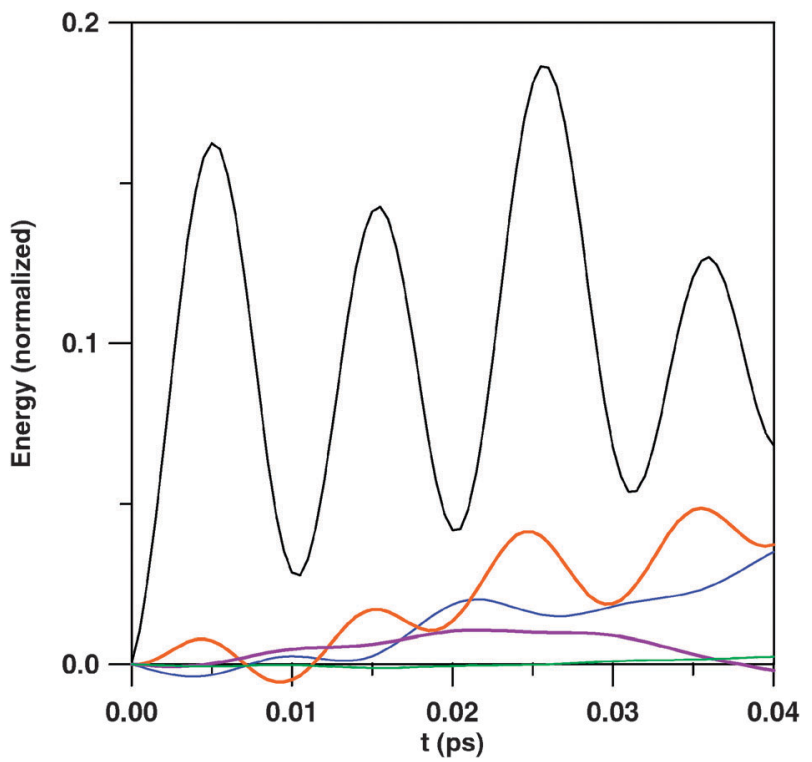

Fig. 2 Short time blow up of energy balance. The same colors as in Fig. 1.

increase in potential energy. Moreover, it is noteworthy that $\Delta V$ 's decrease is the slowest of all the transient contributions, and crosses the vibrational energy relaxation energy at approximately $1.5 \mathrm{ps}$, exhibiting a slowly decaying behavior at longer times. The situation is thus one in which, after all the intramolecular water modes (vibrational, rotational) that have been excited (directly and via Fermi resonance) have relaxed, there is still a feeble signature remaining for the excited water's interaction energy with its neigbors, lasting for a few picoseconds.

\section{E. Hydration shell dependence of the work}

We now address the work performed on translations and rotations as a function of the distance to the initially bendexcited water molecule. To this end, we partition the water molecules surrounding the initially excited molecule in terms of the latter's hydration shells, defined from the oxygen-oxygen radial distribution function, displayed in Fig. 3. The first shell is defined as the one comprising the four closest water molecules at any given instant, a definition which (on the average) corresponds to the first minimum of the radial distribution function (located at $\approx 3.3 \AA$ ); the second shell is defined as consisting of all molecules enclosed within the second minimum ( $\approx 5.7 \AA$ ) excluding those in the first shell; finally, the third shell is bounded by $\approx 5.7 \AA$. While this distance is close to the box size, the use of the Ewald sum allows the determination of contributions from longer distances as well. Fig. 4 displays the results for the work done on rotations for each of the shells just defined (we recall that unity corresponds to the total initial energy). The shape of all the curves is rather similar, but their respective contribution differs widely: work on the four first hydration shell water molecules accounts for almost $60 \%$ of the total work on rotation, that on the water molecules in the second shell represents roughly $35 \%$, while the third shell and beyond account for $4 \%$ and $2 \%$, respectively. Clearly, most of the energy is transferred locally. Although the total work on translation is minimal $(\approx 5 \%)$ the pattern is even more clearcut than for the work on rotation, as shown in Fig. 5; here almost

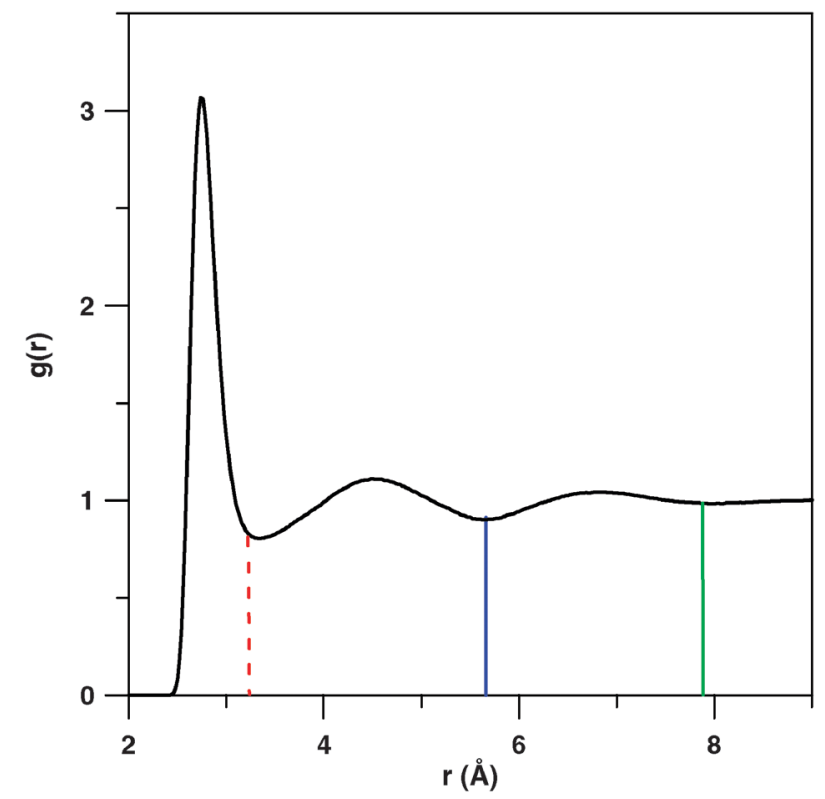

Fig. 3 Oxygen-oxygen radial distribution function. Vertical colored lines define the limits of each hydration shell. The dashed line for the first shell indicates that it is defined from the four closest water molecules at each instant (which on average results in the distance denoted by red vertical bar).

all energy transfer is into the four first hydration shell molecules, with transfer into the second shell and beyond being compatible with zero (the results are subject to considerable statistical noise, given the tiny contribution of translation).

Given the dominance of the first hydration shell for the work on rotation and translation, it is of interest to ascertain the participation of each of the water molecules involved.

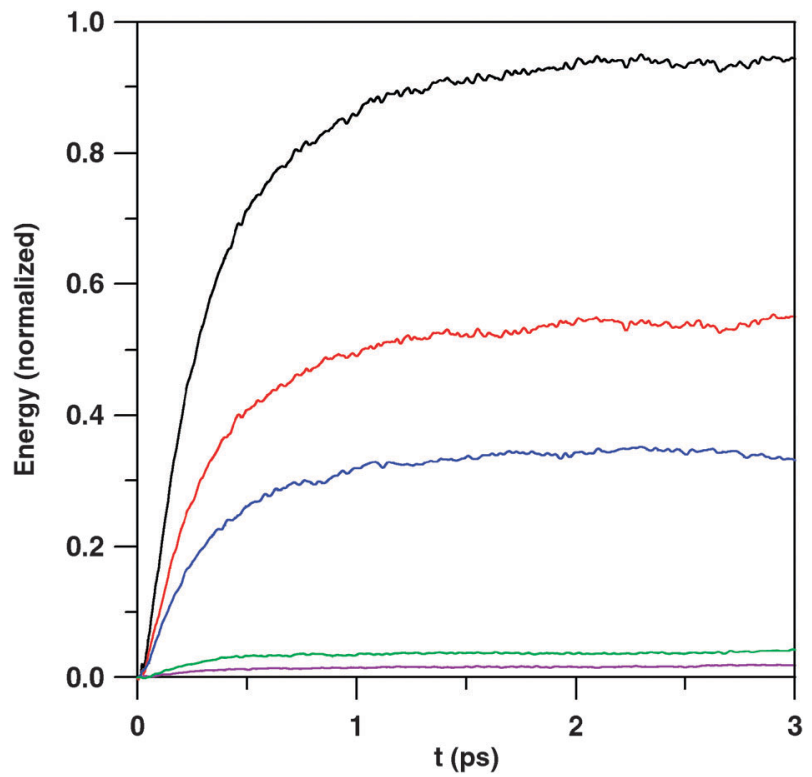

Fig. 4 Work (normalized) on rotational motion of water molecules within each hydration shell. Black line: total work on rotation; red: work on rotation of four closest molecules; blue: on the second hydration shell; green: on the third hydration shell; purple: on molecules beyond the third shell. 


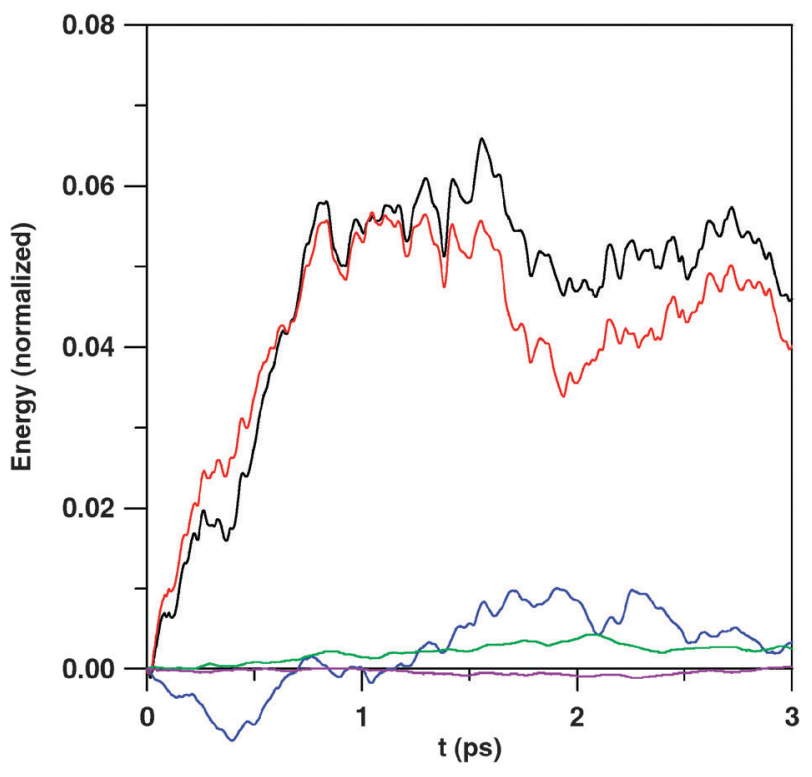

Fig. 5 Work (normalized) on translational motion of water molecules within each hydration shell. Black line: total work on translation; red: work on translation of four closest molecules; blue: on the second hydration shell; green: on the third hydration shell; purple: on molecules beyond the third shell.

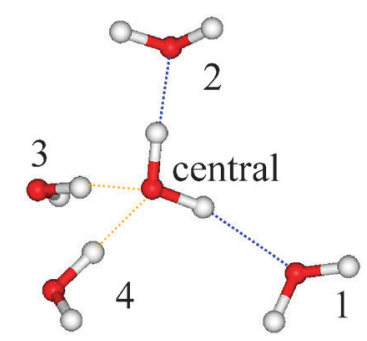

Fig. 6 Sketch of the numbering of molecules around the "central" (bend excited) water molecule. The two water molecules directly H-bonded (blue dotted lines) to the hydrogens of the excited water molecule are referred as 1 and 2, while the other two waters donating H-bonds (orange dotted lines) to the excited water molecule are referred as 3 and 4 .

To this end, we partition these molecules into two groups: the pair of molecules, denoted 1 and 2 (see Fig. 6) hydrogenbonded to the hydrogens of the central molecule, and those, denoted 3 and 4 , hydrogen-bonded to the oxygen of the central molecule. It is found that work on rotation is almost evenly shared by all four molecules, with approximately $56 \%$ of it exerted on molecules 1 and 2, and the rest exerted on molecules 3 and 4, as shown in Fig. 7. This result is consistent with related results reported in ref. 17: it was shown that most of the energy channeled by Fermi resonance excited self-rotation is transferred to molecules 3 and 4 , while that directly transferred from the central water's excited bend into external water molecules is taken up by molecules 1 and 2 . Therefore, given that here we are dealing with the total energy transferred into its neighbors, the addition of both pathways results in that all four neighbors receive a similar flux of energy. In contrast, the work on translation (not shown), and which we recall was mainly done on the first shell, is basically exerted on molecules 1 and 2, with that on molecules 3 and 4 being compatible with zero.

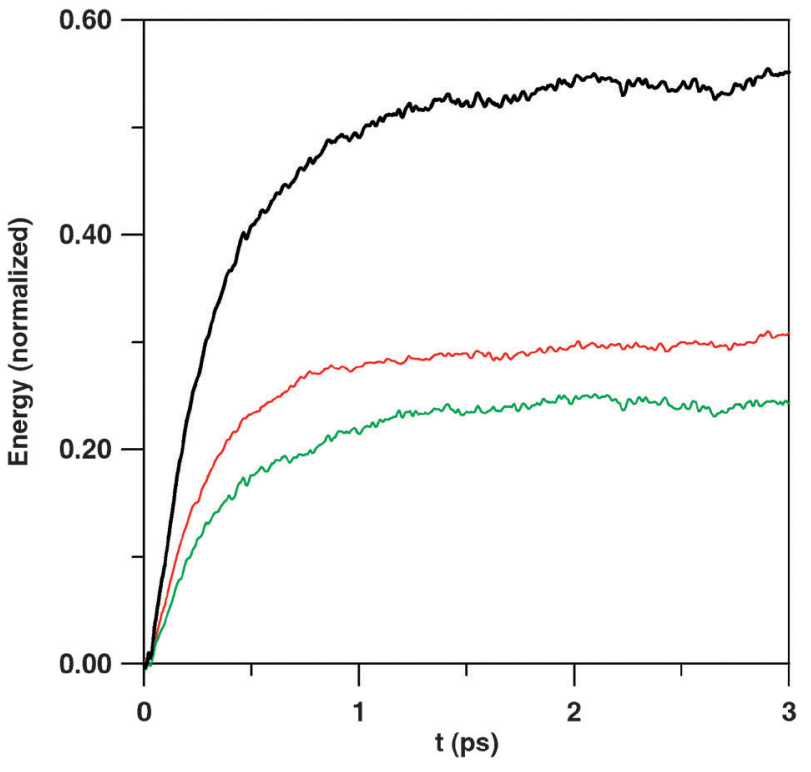

Fig. 7 Work (normalized) on rotational motion of water molecules within the first hydration shell. Black line: total work on the first hydration shell; red: work on molecules 1 and 2 (those hydrogenbonded to the hydrogens of the initially bend-excited molecule); green: the same for molecules 3 and 4 .

\section{F. Temperature dependence}

Recent experiments ${ }^{32}$ have addressed the effect of temperature on vibrational relaxation, with the main finding being an inverse behavior, i.e. bend relaxation slows down with increasing temperature. We have performed a preliminary exploration of this issue, running simulations similar to the ones previously discussed at a temperature of $360 \mathrm{~K}$. It is indeed found that relaxation is slower ( $285 \mathrm{fs} v s .265 \mathrm{fs}$ at $300 \mathrm{~K}$ ), so that the present model captures this effect as well. It must be said, though, that the slowdown is substantially smaller than found experimentally ( $174 \mathrm{fs}$ at $295 \mathrm{~K}$, vs. 250 fs at 348 K). From our calculations (see Fig. 8) it seems that both the intramolecular and intermolecular mechanisms are slightly less efficient during the initial stages, so the slowdown could not be attributed to a single mechanism. In addition, at longer times the intramolecular mechanism slightly diminishes its weight compared to the intermolecular one, although not changing the overall behavior (centrifugal coupling dominating vibrational relaxation). In order to explain this behavior we first note that, for the intramolecular mechanism, hydrogen bond weakening upon temperature increase will cause a downshift of librational overtone frequencies of the excited molecule, and therefore a less optimal overlap with the bending frequency (see for instance Fig. 11 and 17 in ref. 17), although it is also true that the bend frequency also decreases slightly with temperature. A similar phenomenon might be at work for the intermolecular mechanism: while higher frequencies will be more populated, a redshift of water librational frequencies of the solvent will cause a less optimal transfer. From the long time behaviour found in our simulations, it would seem like this latter effect is less important. Given the subtle balance of effects just decribed, and the fact that the model does not fully capture the rate of temperature variation, we do not pursue a further scrutiny at present, since this issue should probably be addressed with a more accurate model. 


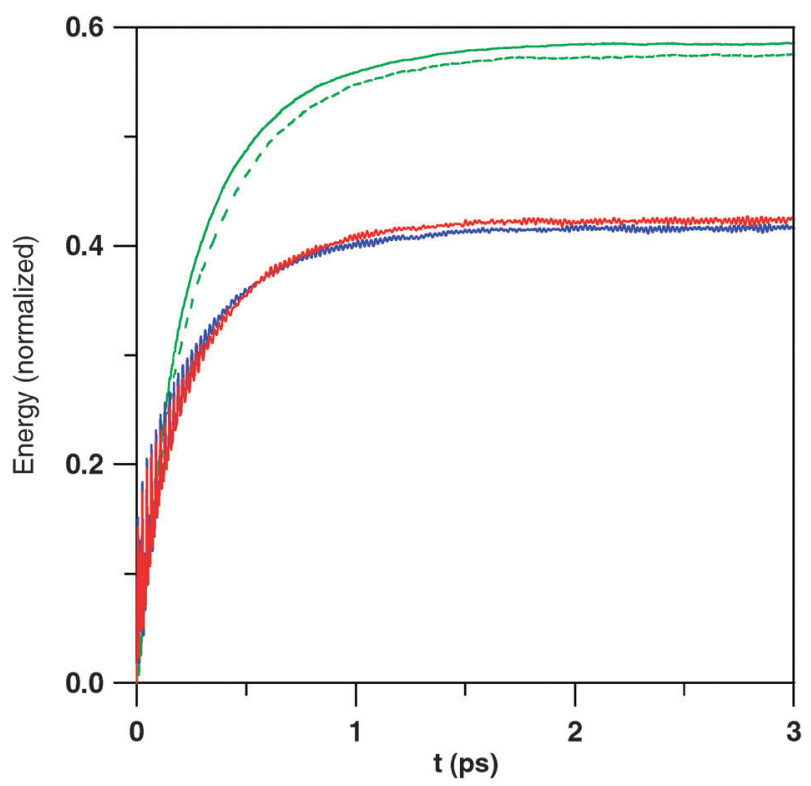

Fig. 8 Comparison of water bend vibrational relaxation mechanisms at different temperatures. Solid green line: vibration to self-rotation transfer at $300 \mathrm{~K}$; dashed green line: the same at $360 \mathrm{~K}$; blue line: direct transfer to neighbors at $300 \mathrm{~K}$; red line: the same at $360 \mathrm{~K}$.

\section{G. Initial conditions and bend energy transfer}

Before we summarize the results of this section, we need to address some aspects related to the generation of initial conditions for the nonequilibrium trajectories reported within. As mentioned in the Computational Details section, there are some differences in the present approach compared to our previous contributions, ${ }^{16,17}$ particularly concerning the generation of the water bending angle at the initial time. Here, bending motion is allowed (during the long equilibration run) for the water molecule that will be subject to vibrational excitation, while in our previous works all molecules were kept rigid. A trivial advantage of this new procedure is that one does not need to sample an initial angle ${ }^{17}$ to start a nonequilibrium trajectory; here we simply add vibrational kinetic energy to a given configuration, up to the total bend vibrational energy.

The basic reason to change to the present procedure, though, is a different one, the small but noticeable effect that angle sampling can have on the behavior of potential energy of interaction change $\Delta V$, one of the basic functions monitored in this work (see e.g. Fig. 1). This subtle effect can be traced back (as explained below) to the fact that the bend angle for the $\mathrm{SPC} / \mathrm{E}$ water model is $109^{\circ}$ while that for a molecule in which bending is allowed is $\approx 105^{\circ}$ : while the shape of the angle distribution obtained here (from which results the mean angle just referred to) and the one used in ref. 17 (corresponding to one of the three different sets examined there, referred to as "thermal distribution") are identical, the position of the peak differs (see ESI $\dagger$ ). In ref. 17's procedure, each nonequilibrium run was started from a configuration obtained with a rigid molecule simulation, so that the surrounding water molecules are initially equilibrated to the aforementioned SPC/E angle of the central water; we denote the mean potential interaction energy in this case as $\langle V\rangle_{109^{\circ}}$. When the relaxation process for the now free to bend central water is complete, the surrounding water molecules will have equilibrated to a smaller angle, with

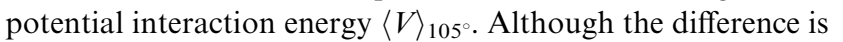
small, $\langle V\rangle_{109^{\circ}}$ is slightly larger, due to the fact that the SPC/E angle is not the optimal one for a flexible molecule. In consequence, at long times one obtains $\langle\Delta V(t)\rangle \rightarrow\langle V\rangle_{105^{\circ}-}$ $\langle V\rangle_{109^{\circ}} \leq 0$, so that the potential energy increment does not tend to zero as required, a behaviour which was not noticed previously since this function was not required in ref. 17 .

This artifact is removed by the simple equilibration procedure followed in the present work, which assures that $\langle\Delta V(t)\rangle \rightarrow$ $\langle V\rangle_{105^{\circ}}-\langle V\rangle_{105^{\circ}}=0$. Since we are now starting each configuration from a more optimal configuration (in what concerns solute-solvent interaction), the present procedure has an impact on the balance of the intermolecular and intramolecular mechanisms that channel the initial water bend excitation energy, and transfer to neighboring molecules is slightly more favored than it was in the description of ref. 17. The quantitative conclusions of ref. 17 are only slightly altered though: the weight of intramolecular transfer to self-rotation (centrifugal mechanism) is reduced from $2 / 3(66 \%)$ of the initial excess water bend energy to about $3 / 5$ (59\%, see Fig. 8) and, correspondingly, intermolecular transfer from the excited water molecule to the surrounding water molecules is increased from $1 / 3$ of the initial excess energy to roughly $2 / 5$. No appreciable differences are found with the overall vibrational relaxation time reported here and previously. ${ }^{16}$

\section{H. Synthesis for water bend energy relaxation}

By merging the present results with those in ref. 16 and 17, we can provide a rather detailed picture of the relaxation process following excitation of the bending motion of a given water molecule in liquid water. First, as was already established in ref. 17, one can distinguish two different channels for the initial decrease in bend vibrational energy:

- An intramolecular transfer into hindered self-rotation via a 2 : 1 Fermi resonance resulting from centrifugal coupling, which dominates the process. Our present estimation, refined by an improved initial condition sampling (Section III G), is that roughly $3 / 5$ of the initial energy is channeled through this mechanism. Most of this energy is taken up by rotational motion around the principal axis parallel to the $\mathrm{HH}$ direction, which has the lowest moment of inertia, and therefore the highest librational frequency. ${ }^{17}$ This energy is then quickly transferred, with a relaxation time $\approx 50 \mathrm{fs}$, into immediate neighbors. Approximately half of this energy is transferred to the four immediate neighbors that constitute the first hydration shell (preferentially to the two water molecules hydrogen bonded to the oxygen of the excited molecule - molecules 3 and 4 in Fig. 6), while the other half is transferred to outer shells. It has been shown here (Section III E) that this energy appears as hindered rotational motion of the energy-accepting waters.

- A direct, intermolecular transfer into neighboring water molecules accounts for $2 / 5$ of the initial water bend excitation energy. Again, most of this energy (approximately two thirds) is transferred to the first hydration shell, although now it is molecules 1 and 2 in Fig. 6 which mainly channel it. Direct transfer to outer shells is thus less important than the one found for self-rotation. It has been shown here (Section III B) 
that this energy appears as hindered rotational motion of the energy-accepting waters.

The combined effect of these two processes results in an overwhelming transfer of energy into hindered rotational motion of its neighbors. We find that roughly $95 \%$ of the energy is transferred into librations, with the rest being transferred into translational motions (see Fig. 1). While we have implicated transfer into librations previously, ${ }^{17}$ as also had been suggested in the experimental studies, ${ }^{14}$ we believe this is the first time in which it is shown unambiguously. In addition, this transfer is rather limited in its spatial extension: roughly $60 \%$ of the energy is transferred into the four immediate water neighbors, and almost all energy is transferred to the two first hydration shells. Direct transfer to molecules beyond two molecular diameters is practically inexistent (see Fig. 4 and 5). Accordingly, one could sum up the present results by stating that water bend vibrational relaxation is rather local, being limited to the two first hydration shells and proceeding through transfer to local librations of the very same molecule at the initial stage and of close neighbors immediately after. Since most of the energy is initially transferred into self-rotation, and the process is basically rotational relaxation after this step, this suggests that a similar picture might also apply to pure rotational relaxation. ${ }^{23}$

A question not addressed here is whether the rapid librational excitation and relaxation would have consequences in experimental anisotropy measurements. Available experimental evidence ${ }^{14}$ suggests that the answer is no, but direct simulation of experimental measures would nonetheless be useful, if only to indicate why there appears to be no effect. A related question which deserves attention is whether rotational excitation would have any significant impact on the jump mechanism/ timescale of water reorientation. ${ }^{34}$ Both these issues are left for future investigation.

In line with the feeble participation of translational motions of the exited water's neighbors in the energy flow, it is also found that transfer to self-translation, mediated by indirect interaction via potential energy with its neighbors, is nil (see Fig. 1). In comparison, the potential energy-due to its important role in mediating intermolecular transfer-shows a considerable increase of up to a fifth of the total initial energy. In addition, it decays slowly, lasting after the excess internal molecular energy has fully decayed (see Fig. 1 and 2).

\section{Concluding remarks}

Via an extension and reformulation in terms of Poisson brackets of the power and work methodology developed previously, ${ }^{17}$ we have explicitly shown that bend vibrational energy of an excited water molecule in liquid water is ultimately transferred to the hindered rotational (librational) motion of its neighbors in the first two hydration shells. Since Section III H has provided a perspective of these new results within the context of the complete picture of the water bend relaxation, we devote the remainder of this section to indicating how the basic formulation can be exploited for other relaxation problems.

The basic Poisson bracket formalism we have developed within can be used to study energy transfer paths for molecular excitations other than the vibrational (bending) one studied here. Two illustrations based on eqn (21) (or its alter ego eqn (22)) will suffice to expose the central ideas. For a purely rotational excitation of a molecule, and assuming that no high frequency vibration of the molecule is excited $\left(\Delta E_{\mathrm{v}}=0\right.$, rigid molecule approximation), rearrangement of eqn (21) gives

$$
\Delta K_{\mathrm{R}}=-\Delta V-\Delta K_{\mathrm{T}}-\sum_{j>1} W_{1 j}
$$

in which case there are transients in the energy transferred to the center of mass kinetic energy $\left(\Delta K_{\mathrm{T}}\right)$ of the molecule and the intermolecular potential energy of interaction with the molecule's neighbors $(\Delta V)$. The remaining work terms can be partitioned, as in e.g. eqn (26), into contributions for the librational and translational motions of the surrounding molecules. The application of this formula to rotational excitation in pure water will be reported elsewhere. ${ }^{23}$

The same approach can be used for solvation energy relaxation. In this scenario, it is the interaction potential energy of the central molecule which is initially subject to a nonequilibrium perturbation, as for instance by means of a photoinduced electronic transition which results in a nonequilibrium solvation energy, studied via time-dependent fluorescence experiments and via simulation. ${ }^{33}$ Again ignoring the excited molecule's vibrational energy for simplicity, rearrangement of eqn (21) gives

$$
\Delta V=-\Delta K_{\mathrm{R}}-\Delta K_{\mathrm{T}}-\sum_{j>1} W_{1 j}
$$

from which one can determine to which solvent neighbors the excess energy is transferred. Note that, as opposed to the situation for the kinetic energy discussed in our first illustration, it is now the averaged kinetic energy increments that will decay to zero after a transient. This solvation dynamics problem will be analyzed in a separate contribution. ${ }^{24}$

\section{Appendix}

Eqn (26) for the water bend relaxation has been derived starting from the time derivative of $H_{\mathrm{mol}}$ for convenience (eqn (17)); one could also start from the time derivative of $H_{\mathrm{v}}($ eqn $(7))$, i.e.

$$
\begin{aligned}
\frac{\mathrm{d} H_{\mathrm{v}}}{\mathrm{d} t} & =\left[H_{\mathrm{v}}, H\right]=\left[H_{\mathrm{v}}, K_{\mathrm{R}}\right]+\left[H_{\mathrm{v}}, V\right] \\
& =\left[H_{\mathrm{v}}, K_{\mathrm{R}}\right]-\frac{\mathrm{d} V}{\mathrm{~d} t}+\left[V, H_{2}\right]+\left[V, K_{\mathrm{R}}\right]+\left[V, K_{\mathrm{T}}\right],
\end{aligned}
$$

which reverts to eqn (12) if we consider that for translation we have generally

$$
\frac{\mathrm{d} K_{\mathrm{T}}}{\mathrm{d} t}=\left[K_{\mathrm{T}}, H\right]=\left[K_{\mathrm{T}}, V\right],
$$

i.e. the only channel is that of external, intermolecular forces, while for rotation

$$
\frac{\mathrm{d} K_{\mathrm{R}}}{\mathrm{d} t}=\left[K_{\mathrm{R}}, H\right]=\left[K_{\mathrm{R}}, H_{\mathrm{v}}\right]+\left[K_{\mathrm{R}}, V\right],
$$

i.e. there are two channels in this case: the internal, intramolecular flux plus the external, intermolecular torques. Finally, upon substituting eqn (32) and (33) into eqn (31), 
and after integration, one recovers eqn (26). This more abstract approach will now be generalized.

We consider the relaxation of an internal mode coupled to several other internal modes and to the environment of the molecule, with Hamiltonian

$$
H=H_{1}+H_{\text {int }}+V+H_{2},
$$

where $H_{1}$ denotes the Hamiltonian for the internal intramolecular mode of interest (bending mode in our case), and $H_{\text {int }}$ the remaining internal Hamiltonian (like rotational/ translational motions in our case). We will now consider three alternative expressions for the power on mode 1 , each one providing information on different aspects of the process.

First, we consider the time evolution of the three first terms

$$
\begin{gathered}
\frac{\mathrm{d} H_{1}}{\mathrm{~d} t}=\left[H_{1}, H_{\text {int }}\right]+\left[H_{1}, V\right], \\
\frac{\mathrm{d} H_{\text {int }}}{\mathrm{d} t}=\left[H_{\text {int }}, H_{1}\right]+\left[H_{\text {int }}, V\right], \\
\frac{\mathrm{d} V}{\mathrm{~d} t}=\left[V, H_{1}\right]+\left[V, H_{\text {int }}\right]+\left[V, H_{2}\right] .
\end{gathered}
$$

The first equation describes the balance of energy fluxes for the mode of interest: $\left[H_{1}, H_{\text {int }}\right]$ represents the flux to other internal modes (which can be partitioned into different contributions by the Poisson bracket's additive structure, see e.g. eqn (11)), and $\left[H_{1}, V\right]$ corresponds to the power exerted by the environment (which again can also be partitioned into contributions from different neighbors). Eqn (35) was employed in ref. 17, in the expanded form found in eqn (12).

A second expression for $\mathrm{d} H_{1} / \mathrm{d} t$ is obtained if the $\left[H_{1}, V\right]$ term in eqn (35) is rewritten in terms of the potential energy change, with the purpose of ascertaining the specific accepting mode. Substituting $\mathrm{d} V / \mathrm{d} t$ (eqn (37)) into the first equation we obtain

$$
\begin{gathered}
\frac{\mathrm{d} H_{1}}{\mathrm{~d} t}=\left[H_{1}, H_{\text {int }}\right]-\frac{\mathrm{d} V}{\mathrm{~d} t}+\left[V, H_{2}\right]+\left[V, H_{\text {int }}\right], \\
\frac{\mathrm{d} H_{\text {int }}}{\mathrm{d} t}=\left[H_{\text {int }}, H_{1}\right]+\left[H_{\text {int }}, V\right] .
\end{gathered}
$$

Eqn (38) provides a more detailed view of all the energy fluxes in which the excited mode (1) is involved. The last three terms, which add up to the entire work performed by the environment, can be interpreted as: (a) energy in the excited mode converted into an intermolecular potential energy increment $(\mathrm{d} V / \mathrm{d} t)$; (b) power exerted on the environment modes $\left(\left[\mathrm{V}, \mathrm{H}_{2}\right]\right)$, i.e. the part that we are interested in; (c) work on the rest of the molecule's internal modes of the molecule $\left(\left[H_{\mathrm{int}}, V\right]\right.$ term). This last term shows how transfer of energy between the excited mode and the internal modes can be indirect through this intermolecular term, in addition to the direct intramolecular route $\left(\left[H_{1}, H_{\text {int }}\right]\right)$.

These two contributions (direct and indirect) can be found again within the second equation (eqn (39)), which represents the time evolution of energy contained in the rest of internal molecular modes. Of course this time both contributions come with the opposite sign (compared to eqn (38)). In addition, while they both contribute in general, the first one $\left(\left[H_{\mathrm{int}}, H_{1}\right]\right)$ vanishes for instance in the case of (rigid molecule) rotational relaxation (as there is no direct coupling with the other internal mode, namely center of mass translation). If one is also interested in the time evolution of other internal modes, $H_{\text {int }}$ could be partitioned in as many contributions as required, so that eqn (39) would branch into a number of similar equations. We thus see that the set eqn (38) and (39) allows a detailed monitoring of energy fluxes during relaxation of the excited mode.

Finally, a third expression can be obtained for $\mathrm{d} H_{1} / \mathrm{d} t$ if one is not interested in the specific energy fluxes between the excited mode and the remaining internal modes. Combining eqn (38) and (39), we find the generalization of eqn (26)

$$
\frac{\mathrm{d} H_{1}}{\mathrm{~d} t}=\left[V, H_{2}\right]-\frac{\mathrm{d} V}{\mathrm{~d} t}-\frac{\mathrm{d} H_{\mathrm{int}}}{\mathrm{d} t},
$$

which in a way summarizes the whole process. If one thinks in terms of an excited mode (1), the change in its excess energy ( $\left.\mathrm{d} H_{1} / \mathrm{d} t\right)$ is transferred into: (a) environment degrees of freedom $\left(\left[\mathrm{V}, \mathrm{H}_{2}\right]\right)$; (b) potential interaction energy $(\mathrm{d} V / \mathrm{d} t)$; and (c) internal modes of the solute $\left(\mathrm{d} H_{\text {int }} / \mathrm{d} t\right)$, discussed above in terms of direct and indirect channels.

If applied to a case in which the non-initially excited modes are thermalized at the beginning and end of the process, then $\langle\Delta V\rangle,\left\langle\Delta H_{\text {int }}\right\rangle \rightarrow 0$. Therefore, after integration one obtains

$$
\left\langle\Delta H_{1}\right\rangle \rightarrow\left\langle\int_{0}^{\infty}\left[V, H_{2}\right] \mathrm{d} t\right\rangle=\left\langle\int_{0}^{\infty} \frac{\partial V}{\partial q_{i}} \frac{\partial H_{2}}{\partial p_{i}} \mathrm{~d} t\right\rangle,
$$

which allows one to ascertain, by partitioning into different contributions (see eqn (23)), the percentage of excess molecular energy that has been channeled (on the average) through each specific solvent mode.

\section{Acknowledgements}

This work was supported by M.C.I. FIS2009-13641-C02-01, D.G.R. 2009SGR-1003 (R.R.) and by NSF grants CHE-0750477 and CHE-1112564 (J.T.H.)

\section{References}

1 A. Laubereau and W. Kaiser, Rev. Mod. Phys., 1978, 50, 607.

2 D. W. Oxtoby, Adv. Chem. Phys., 1979, 40, 1; D. W. Oxtoby, Adv. Chem. Phys., 1981, 47, 487; D. W. Oxtoby, Annu. Rev. Phys. Chem., 1981, 32, 77.

3 J. Chesnoy and G. M. Gale, Adv. Chem. Phys., 1988, 70, 297.

4 C. B. Harris, D. E. Smith and D. J. Russell, Chem. Rev., 1990, 90, 481.

5 J. C. Owrutsky, D. Raftery and R. M. Hochstrasser, Annu. Rev. Phys. Chem., 1994, 45, 519.

6 J. T. Hynes and R. Rey, in Infrared and Raman Spectroscopy, ed. M. D. Fayer, Marcel Dekker, New York, 2001.

7 D. D. Dlott, Chem. Phys., 2001, 266, 149.

8 R. Rey, K. B. Moller and J. T. Hynes, Chem. Rev., 2004, 104, 1915.

9 E. L. Sibert, S. G. Ramesh and T. S. Gulmen, J. Phys. Chem. A, 2008, 112, 11291.

10 J. L. Skinner, Mol. Phys., 2008, 106, 2245.

11 J. L. Skinner, Theor. Chem. Acc., 2011, 128, 147.

12 E. T. J. Nibbering and T. Elsaesser, Chem. Rev., 2004, 104, 1887.

13 R. Rey and J. T. Hynes, J. Chem. Phys., 1996, 104, 2356.

14 S. Ashihara, N. Huse, E. T. J. Nibbering and T. Elsaesser, Chem. Phys. Lett., 2006, 424, 66; N. Huse, S. Ashihara, E. T. J. Nibbering and T. Elsaesser, Chem. Phys. Lett., 2005, 404, 389; S. Ashihara, 
N. Huse, A. Espagne, E. T. J. Nibbering and T. Elsaesser, J. Phys. Chem. A, 2007, 115, 743 .

15 M. Buchner, B. M. Ladanyi and R. M. Stratt, J. Chem. Phys., 1992, 97, 8522.

16 F. Ingrosso, R. Rey, T. Elsaesser and J. T. Hynes, J. Phys. Chem. $A, 2009,113,6657$.

17 R. Rey, F. Ingrosso, T. Elsaesser and J. T. Hynes, J. Phys. Chem. $A, 2009, \mathbf{1 1 3}, 8949$.

18 (a) R. M. Whitnell, K. R. Wilson and J. T. Hynes, J. Phys. Chem., 1990, 94, 8625; (b) R. M. Whitnell, K. R. Wilson and J. T. Hynes, J. Chem. Phys., 1992, 96, 5354.

19 H. Goldstein, Classical Mechanics, Addison-Wesley, Reading, MA, 1980.

20 J. C. Wilson and P. C. Decius, Molecular Vibrations, Dover, New York, 1980.

21 R. Rey, Chem. Phys., 1998, 229, 217.

22 J. B. Marion and S. T. Thornton, Classical Dynamics of Particles and Systems, Harcourt Brace Jovanovich, Orlando, 1988.

23 J. Petersen, K. B. Moller, R. Rey and J. T. Hynes, to be submitted.

24 R. Rey and J. T. Hynes, work in progress.

25 H. J. C. Berendsen, J. R. Grigera and T. P. J. Straatsma, J. Phys. Chem., 1987, 91, 6269.

26 H. C. Andersen, J. Comput. Phys., 1983, 52, 24.

27 S. G. Ramesh and E. L. Sibert, J. Chem. Phys., 2006, 125, 244516.

28 S. C. Harvey, R. K. Z. Tan and T. E. Cheatham III, J. Comput. Chem., 1998, 19, 726.

29 M. Lingenheil, R. Denschlag, R. Reichold and P. Tavan, J. Chem. Theory Comput., 2008, 4, 1293.

30 H. J. C. Berendsen, J. P. M. Postma, W. F. van Gunsteren, A. DiNola and J. R. Haak, J. Chem. Phys., 1984, 81, 3684.
31 Other infrared experiments give slightly longer times ranging up to 260 fs, see, e.g.: (a) J. Lindner, P. Vohringer, M. S. Pshenichnikov, D. Cringus, D. A. Wiersma and M. Mostovoy, Chem. Phys. Lett., 2006, 421, 329; (b) J. Lindner, D. Cringen, M. S. Pshenichnikov and P. Vohringer, Chem. Phys., 2007, 341, 326; (c) C. H. Kuo and R. M. Hochstrasser, Chem. Phys., 2007, 341, 21; (d) L. Chieffo, J. Shattuck, J. J. Amsden, S. Eramilli and L. D. Ziegler, Chem. Phys., 2007, 341, 71. These references deal with a bend librational combination band.

32 S. Ashihara, S. Fujioka and K. Shibuya, Chem. Phys. Lett., 2011, 502, 57.

33 (a) G. van der Zwan and J. T. Hynes, J. Phys. Chem., 1985, 89, 4181; (b) E. A. Carter and J. T. Hynes, J. Chem. Phys., 1991, 94, 5961; (c) M. Maroncelli and G. R. Fleming, J. Chem. Phys., 1988, 89, 5044; (d) M. Maroncelli, J. Mol. Liq., 1993, 57, 1; (e) R. Jimenez, G. R. Fleming, P. V. Kumar and M. Maroncelli, Nature, 1994, 369, 471; (f) J. Gardecki, M. L. Horng, A. Papazyan and M. Maroncelli, Stud. Phys. Theor. Chem., 1995, 83, 49; (g) G. R. Fleming and M. H. Cho, Annu. Rev. Phys. Chem., 1996, 47, 109; $(h)$ W. P. de Boeij, M. S. Pshenichnikov and D. A. Wiersma, Аnnu. Rev. Phys. Chem., 1998, 49, 99; (i) N. Nandi, K. Bhattacharyya and B. Bagchi, Chem. Rev., 2000, 100, 2013; (j) B. Bagchi and B. Jana, Chem. Soc. Rev., 2010, 39, 1936; (k) K. B. Eisenthal, Chem. Rev., 2006, 106, 1462; (l) W. H. Thompson, Annu. Rev. Phys. Chem., 2011, 62, 599.

34 (a) D. Laage, G. Stirnemann, F. Sterpone, R. Rey and J. T. Hynes, Annu. Rev. Phys. Chem., 2011, 62, 395; (b) D. Laage and J. T. Hynes, Science, 2006, 311, 832; (c) M. B. Ji, M. Odelius and K. J. Gaffney, Science, 2010, 328, 1003; (d) S. Park, M. Odelius and K. J. Gaffney, J. Phys. Chem. B, 2009, 113, 7825. 